\title{
ON THE CONGRUENCE LATTICES OF UNARY ALGEBRAS
}

\author{
JOEL BERMAN
}

\begin{abstract}
Characterizations of those unary algebras whose congruence lattices are semimodular or atomic are obtained. Combining these results gives necessary and sufficient conditions for a unary algebra to have a geometric congruence lattice.
\end{abstract}

A unary algebra, $\mathfrak{U}=\langle A ; f\rangle$, is a set $A$ together with a function $f$ from $A$ into $A$. A congruence relation $\theta$ of $\mathfrak{A}$ is an equivalence relation on $A$ such that if $x \equiv y(\theta)$ then $f(x) \equiv f(y)(\theta)$. The congruence lattice of $\mathfrak{A}$, denoted $\Theta(\mathfrak{U})$, is the collection of all congruence relations of $\mathfrak{A}$ partially ordered in the usual manner [2, p. 50]. If $L$ is a lattice and $a, b \in L$, then $b$ is said to cover $a$ if $a<b$ and $\{x \mid a<x<b\}=\varnothing$. This relation will be denoted $a<b$. $L$ is semimodular if for any $a, b$ and $c \in L$ such that $c<a$ and $c<b$ then $a<a \vee b$ and $b<a \vee b$. Theorem 1 gives a characterization of those unary algebras whose congruence lattices are semimodular. An atom of a lattice with 0 is an element that covers 0 . A lattice is atomic if every element is a join of atoms. Theorem 2 gives a characterization of those unary algebras whose congruence lattices are atomic.

Let $\mathfrak{U}=\langle A ; f\rangle$ be a unary algebra. For any $x \in A$ and any positive integer $n$ let $f^{0}(x)=x$ and $f^{n}(x)=f\left(f^{n-1}(x)\right)$. A component of $\mathfrak{U}$ is a subset of $A$ such that $x, y$ are in the same component if and only if there exist $m, n \in N$ such that $f^{n}(x)=f^{n}(y)$. Index the components of $\mathfrak{A}$ by an index set $I$ so that $A=\bigcup_{i \in I} A_{i}$. For each component $A_{i}$, choose a fixed subset $C_{i}$, called a cycle, as follows: If there exists $n \in N$ and $x \in A_{i}$ such that $f^{n}(x)=x$, let $C_{i}=\left\{f^{k}(x) \mid k \in N\right\}$. Such a cycle is a unique finite subset of $A_{i}$. Denote such a finite cycle by $\left\{x_{0}, x_{1}, \cdots, x_{m-1}\right\}$, with $f\left(x_{i}\right)=x_{i+1}$. If $n$ is any integer let $x_{n}=x_{i}$ where $n \equiv i(\bmod m), 0 \leqq i<m$. If no such $x$ and $n$ exist for $A_{i}$ let $x_{0}$ be any fixed element of $A_{i}, C_{i}=\left\{f^{\prime \prime}\left(x_{0}\right) \mid m \in N\right\}$. Denote this set by $\left\{x_{0}, x_{1}, x_{2}, \cdots\right\}$ where $f\left(x_{i}\right)=x_{i+1}$. If $C_{i}$ and $C_{j}$ are any two cycles, $\left|C_{i}\right|=m_{i}$ and $\left|C_{j}\right|=m_{j}$, then $C_{i}$ and $C_{j}$ are said to have relatively prime cardinalities if both $m_{i}$ and $m_{j}$ are finite and $\left(m_{i}, m_{j}\right)=1$ or if $m_{i}$ is

Received by the editors November 16, 1971 .

AMS 1970 subject classifications. Primary 08A25, 06A20; Secondary 05B35, 94A25.

Key words and phrases. Congruence relations, congruence lattices, unary algebras, universal algebras, semimodular lattices, atomic lattices, matroid lattices, geometric lattices, sequential machines.

(C) American Mathematical Society 1972 
infinite and $m_{j}=1$. A general discussion of unary algebras can be found in [3] and [4].

If $B \subseteq A$, let $\theta(B)$ denote the smallest element of $\Theta(\mathfrak{A})$ for which $B$ is contained in one congruence class. $\theta(\{x, y\})$ is usually denoted by $\theta(x, y)$. $I_{A}$ and $U_{A}$ will denote $\{\langle x, x\rangle \mid x \in A\}$ and $A \times A$ respectively. A congruence class is said to be nontrivial if it has more than one element. Finally, if $L$ is any lattice, $a, b \in L$, then $[a, b]=\{x \mid x \in L$ and $a \leqq x \leqq b\}$.

LEMMA 1. Let $\mathfrak{U}=\langle A ; f\rangle$ and $\mathfrak{B}=\langle B ; g\rangle$ and $\mathfrak{C}=\langle C ; h\rangle$ be unary algebras such that $\mathfrak{B}$ is any homomorphic image of $\mathfrak{U}$ and $\mathfrak{C}$ is any subalgebra of $\mathfrak{A}$. Then $\Theta(\mathfrak{B})$ is isomorphic to a dual ideal of $\Theta(\mathfrak{A})$ and $\Theta(\mathfrak{C})$ is isomorphic to an ideal of $\Theta(\mathfrak{U})$.

Proof. The result for homomorphic images holds for any algebra $[2$, p. 61]. As for subalgebras, since $\mathfrak{U}$ is a unary algebra, $C$ is the only nontrivial congruence class of $\theta(C)$. Hence the interval $\left[I_{A}, \theta(C)\right]$ in $\Theta(\mathfrak{U})$ is isomorphic to $\Theta(\mathbb{C})$.

Lemma 2. Let $C$ be any cycle of some unary algebra $\mathfrak{A}=\langle A ; f\rangle$. Then $\left[I_{A}, \theta(C)\right]$ is semimodular.

Proof. If $C$ is finite then $\left[I_{A}, \theta(C)\right]$ is isomorphic to the lattice of divisors of $|C|$ and is distributive and hence semimodular. If $C$ is infinite and if $\theta \in\left[I_{A}, \theta(C)\right]$ then $\theta=\theta\left(x_{m}, x_{m+p}\right)$ for fixed $m$ and $p$. Also $\theta<\Psi$ if and only if $\Psi=\theta\left(x_{m+1}, x_{m+1+p}\right)$ or $\Psi=\theta\left(x_{m}, x_{m+q}\right)$ where $p / q$ is prime. An examination of these possibilities shows semimodularity holds.

Lemma 3. Let $\mathfrak{U}=\langle A ; f\rangle$ be a unary algebra. Then the atoms of $\Theta(\mathfrak{U})$ are of the following types:

(i) atoms of $\left[I_{A}, \theta\left(C_{i}\right)\right]$ where $C_{i}$ is a finite cycle;

(ii) $\theta(x, y)$ where $f(x)=f(y)$;

(iii) $\theta(x, y)$ where $x \in C_{i}, y \in C_{j}, i \neq j,\left|C_{i}\right|=\left|C_{j}\right|$ and $\left|C_{i}\right|$ is finite.

Proof. Let $\theta$ be an atom, $x \equiv y(\theta), x \neq y$. Then if $\theta$ is not of type (ii) then $\theta=\theta(x, y)=\theta(f(x), f(y))$. This implies that $\{x, y\} \subseteq\left\{f^{n}(x) \mid n \geqq 1\right\} \cup$ $\left\{f^{n}(y) \mid n \geqq 1\right\}$. Therefore $x$ and $y$ must be in finite cycles. If they are in the same cycle (i) must hold. If they are in different cycles (iii) must hold.

THeOREM 1. Let $\mathfrak{U}=\langle A ; f\rangle$ be a unary algebra. Then $\Theta(\mathfrak{U})$ is upper semimodular if and only if the cycles of $\mathfrak{U}$ have relatively prime cardinalities.

Proof. Suppose that the cycles of $\mathfrak{A}$ are of relatively prime cardinality and $\alpha, \beta$, and $\tau$ are in $\Theta(\mathfrak{U}), \tau<\alpha$ and $\tau<\beta$. Let $\overline{\mathfrak{U}}=\mathfrak{U} / \tau$. It follows that the cycles of $\mathfrak{A}$ are also of relatively prime cardinality. Also $\Theta(\overline{\mathfrak{A}}) \cong$ $\left[\tau, U_{A}\right]$ in $\Theta(\mathfrak{U})$ by Lemma 1 . Let $\bar{\alpha}$ and $\bar{\beta}$ be the images of $\alpha$ and $\beta$ in 
$\Theta(\overline{\mathfrak{U}})$. Thus $\bar{\alpha}$ and $\bar{\beta}$ are atoms of $\Theta(\overline{\mathfrak{U}})$. So any atom of type (iii) in Lemma 3 will have only one nontrivial congruence class and this will be of cardinality two.

It will be sufficient to show that $\bar{\beta}<\bar{\beta} \vee \bar{\alpha}$ and $\bar{\alpha}<\bar{\beta} \vee \bar{\alpha}$ in $\Theta(\overline{\mathcal{U}})$. Note that if $\bar{\alpha}$ and $\bar{\beta}$ are such that their nontrivial congruence classes are disjoint then indeed $\bar{\beta}<\bar{\beta} \vee \bar{\alpha}$ and $\bar{\alpha}<\bar{\beta} \vee \bar{\alpha}$. Since $\bar{\beta}$ and $\bar{\alpha}$ are atoms and by Lemma 3 there are only three types of atoms, there are essentially only six different cases to consider:

$\bar{\alpha}$ and $\bar{\beta}$ both of type (i). Let $\bar{\alpha} \leqq \theta\left(C_{i}\right)$ and $\bar{\beta} \leqq \theta\left(C_{j}\right)$ for cycles $C_{i}$ and $C_{j}$ in $\mathfrak{\mathcal { A }}$. By Lemma 2 it can be assumed that $i \neq j$. Also

$$
\bar{\alpha} \vee \bar{\beta} \in\left[I_{A}, \theta\left(C_{i}\right) \vee \theta\left(C_{j}\right)\right] \simeq\left[I_{A}, \theta\left(C_{i}\right)\right] \times\left[I_{A}, \theta\left(C_{j}\right)\right] .
$$

From Lemma 2 and the fact that the product of semimodular lattices is semimodular, semimodularity holds in this case.

$\bar{\alpha}$ of type (i) and $\bar{\beta}$ of type (ii). Let $\bar{\alpha} \leqq \theta\left(C_{i}\right)$ and $\bar{\beta}=\theta(x, y)$ where $f(x)=f(y) . \bar{\beta}$ has only one nontrivial congruence class and this will be of cardinality two. By the remarks above concerning disjoint congruence classes it can be assumed that $x \in C_{i}$ and $y \notin C_{i}$. But then $\bar{\beta} \vee \bar{\alpha}$ has as its congruence classes those of $\bar{\alpha}$ with $y$ adjoined to one of them. Clearly then $\bar{\beta}<\bar{\beta} \vee \bar{\alpha}$ and $\bar{\alpha}<\bar{\beta} \vee \bar{\alpha}$.

$\bar{\alpha}$ of type (i) and $\bar{\beta}$ of type (iii). Since the cycles of $\overline{\mathfrak{U}}$ are of relatively prime cardinalities, $\bar{\alpha}$ and $\bar{\beta}$ must have disjoint nontrivial congruence classes so the desired covering relations hold. The remaining three cases are handled similarly.

Conversely, it will be shown that the interval sublattice $\left[I_{A}, \theta\left(C_{i} \cup C_{j}\right)\right]$ is not semimodular if the cardinalities of $C_{i}$ and $C_{j}$ are not relatively prime. Let $\left|C_{i}\right|=m_{i}$ and $\left|C_{j}\right|=m_{j}$. If $m_{i}$ and $m_{j}$ are both finite let $p$ be any prime that divides both of them. If only $m_{i}$ is finite let $p$ be any prime that divides $m_{i}$. If $m_{i}$ and $m_{j}$ are both infinite let $p$ be any prime. Let $\left\{x_{0}, x_{1}, x_{2}, \cdots\right\} \subseteq C_{i}$ and $\left\{y_{0}, y_{1}, y_{2}, \cdots\right\} \subseteq C_{j}$ where $f\left(x_{t}\right)=x_{t+1}$ and $f\left(y_{t}\right)=y_{t+1}$. Let $\tau \in \Theta(\mathfrak{U})$ be $\tau=\theta\left(x_{0}, y_{0}, x_{p}, y_{p}\right)$. Since $p$ is a prime $\tau<\theta\left(x_{0}, y_{0}, x_{1}, y_{1}\right)$. Also $\left(\tau \wedge\left(\theta\left(x_{0}, x_{1}\right) \vee \theta\left(y_{0}, y_{1}\right)\right)\right)=\theta\left(x_{0}, x_{p}\right) \vee \theta\left(y_{0}, y_{p}\right)<\tau$. Also $\theta\left(x_{0}, x_{p}\right) \vee \theta\left(y_{0}, y_{p}\right)<\theta\left(x_{0}, x_{p}\right) \vee \theta\left(y_{0}, y_{1}\right)$, since $p$ is prime. This gives the following covering diagram:

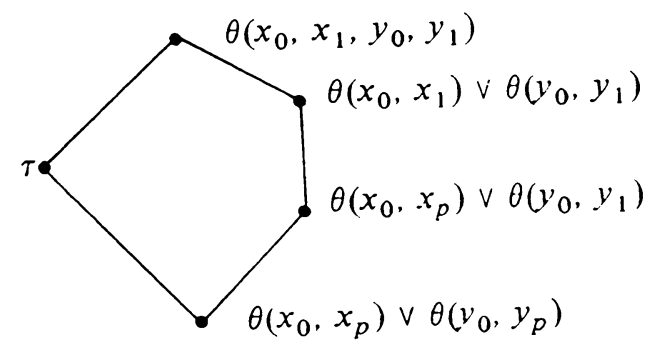


This shows that semimodularity cannot hold in $\left[I_{A}, \theta\left(C_{i} \cup C_{j}\right)\right]$.

Note. If $\mathfrak{U}$ has only one component then $\Theta(\mathfrak{U})$ is semimodular. This gives, in the finite case, Theorem 3 of [6].

THEOREM 2. Let $\mathfrak{A}$ be a unary algebra with components $A_{i}$ and cycles $C_{i}$, $i \in I$. Then $\Theta(\mathfrak{U})$ is atomic if and only if $\mathfrak{A}$ satisfies the following conditions:

(i) $\left|C_{i}\right|$ is finite for all $i \in I$.

(ii) $\left|C_{i}\right|=\left|C_{j}\right|$ for all $i, j \in I$.

(iii) $\left|C_{i}\right|$ is a square free integer for all $i \in I$.

(iv) For at most one $i \in I$ does $A_{i} \neq C_{i}$, in which case there exists $x_{0} \in C_{i}$ such that for all $u \in A_{i}, u \notin C_{i}, f(u)=x_{0}$.

Proof. Let $\mathfrak{U}$ be such that $\Theta(\mathfrak{U})$ is atomic. Then (i) must hold since if $C_{i}$ is an infinite cycle, the interval $\left[I_{A}, \theta\left(C_{i}\right)\right]$ contains no atoms. If $\left|C_{i}\right| \neq\left|C_{j}\right|$ and $x_{0} \in C_{i}$ and $y_{0} \in C_{j}$ then, by Lemma $3, \theta\left(x_{0}, y_{0}\right)$ is not an atom. But $\theta\left(x_{0}, y_{0}\right)$ is also join irreducible. Hence (ii) holds. Next, suppose contrary to (iii) that $\left|C_{i}\right|=p^{2} q$ where $p$ is some prime number. Let $x_{0}, x_{q}$ be in $C_{i}$. The element $\theta\left(x_{0}, x_{q}\right)$ is not an atom in $\Theta(\mathfrak{U})$ since it covers $\theta\left(x_{0}, x_{p q}\right)$. But $\theta\left(x_{0}, x_{q}\right)$ is also join irreducible. This contradicts the fact that $\Theta(\mathscr{U})$ is atomic. Now suppose that there exists $i \neq j$ such that $A_{i} \neq C_{i}$ and $A_{j} \neq C_{j}$. Let $u \in A_{i} \mid C_{i}$ and $v \in A_{j} \mid C_{j}$ be such that $f(u)=x_{0} \in C_{i}$ and $f(v)=y_{0} \in C_{j} . \theta(u, v)$ is not an atom since $\theta(u, v)>\theta\left(x_{0}, y_{0}\right)>I_{A}$. But again $\theta(u, v)$ is join irreducible. This shows that the first part of (iv) must hold. A similar argument applies to $u, v \in A_{i} \mid C_{i}, f(u) \neq f(v), f(u), f(v) \in C_{i}$. Finally, suppose there exists $u \in A_{i} \mid C_{i}, f(u) \notin C_{i}$. Let $f^{n}(u)=x_{0}$, so $n>1$. Consider $\theta\left(u, x_{-n}\right)$; it is also nonatomic and join irreducible. Hence (iv) holds.

Conversely, suppose conditions (i)-(iv) hold. Let $x_{0}$ be as in condition (iv). Form $S=\left\{u \mid f(u)=x_{0}\right\}$. Then $\left[I_{A}, \theta(S)\right]$ is isomorphic to $\mathscr{E}(S)$, the lattice of all equivalence relations on $S$. Since $\mathscr{E}(S)$ is atomic, it follows that $\left[I_{A}, \theta(S)\right]$ is also. Let $\alpha \in \Theta(\mathfrak{R})$. Claim $\alpha=\left(\alpha \wedge \theta\left(\cup C_{i}\right)\right) \vee(\alpha \wedge \theta(S))$. For if $y_{i} \equiv u(\alpha)$ where $y_{i} \in C_{j}$ and $u \notin \cup C_{i}$, then $y_{i+1} \equiv x_{0}(\alpha)$ and hence $y_{i} \equiv x_{-1}(\alpha)$. But then $y_{i} \equiv x_{-1}\left(\alpha \wedge \theta\left(\bigcup C_{i}\right)\right)$ and $y_{i} \equiv x_{-1} \equiv u(\alpha)$ so $x_{-1} \equiv$ $u(\alpha \wedge \theta(S))$ so that $u \equiv y_{i}\left(\alpha \wedge \theta\left(\bigcup C_{i}\right)\right) \vee(\alpha \wedge \theta(S))$. This verifies the claim. Hence all that remains is to show that the interval $\left[I_{A}, \theta\left(\cup C_{i}\right)\right]$ is atomic. Conditions (i) and (iii) imply that $\left[I_{A}, \theta\left(C_{i}\right)\right]$ is atomic and hence $\left[I_{A}, \bigvee_{i \in I} \theta\left(C_{i}\right)\right]$ is also atomic. Condition (ii) guarantees that any element of the form $\theta(x, y)$ is an atom for $x \in C_{k}$ and $y \in C_{j}, k \neq j$. But any element in $\left[I_{A}, \theta\left(\bigcup_{i \in I} C_{i}\right)\right]$ is the join of elements $\theta(x, y)$ where either $x, y \in C_{j}$ or $x \in C_{j}$ and $y \in C_{k}$. But these are either atoms or joins of atoms so the proof is complete.

Definition. A geometric lattice is a finite dimensional lattice that is both atomic and semimodular. 
THEOREM 3. A unary algebra $\mathfrak{U}=\langle A ; f\rangle$ has a congruence lattice which is a geometric lattice if and only if $A$ is finite and

(1) $\mathfrak{U}$ has exactly one cycle $C,|C|$ is square free and for all $x, y \in A \backslash C$, $f(x)=f(y) \in C$.

(2) Every cycle of $\mathfrak{A}$ is of cardinality 1 , and if $C$ is the union of all these cycles, then for all $x, y \in A \backslash C, f(x)=f(y) \in C$.

Proof. Combine the conditions of Theorems 1 and 2.

Note. If the conditions of Theorem 3 hold then it is easily verified that $\Theta(\mathfrak{Q}) \simeq\left[I_{A}, \theta(C)\right] \times\left[I_{A}, \theta(T)\right]$ where $T=\{x \mid f(x)=f(y), x \neq y\}$.

COROLlaRY. Let $L$ be a geometric lattice. Then $L$ is isomorphic to the congruence lattice of some unary algebra if and only if $L \simeq 2^{p} \times \mathscr{E}(S)$ or $L \simeq \mathscr{E}(S) \times \mathscr{E}(T)$, where $p$ is a finite positive integer and $S$ and $T$ are finite sets.

\section{REFERENCES}

1. G. Birkhoff, Lattice theory, 3rd ed., Amer. Math. Soc. Colloq. Publ., vol. 25, Amer. Math. Soc., Providence, R.I., 1967. MR 37 \#2638.

2. G. Grätzer, Universal algebra, Van Nostrand, Princeton, N.J., 1968. MR 40 $\# 1320$.

3. J. Johnson and R. L. Seifert, A survey of multiunary algebras, Mimeographed Seminar Notes (R. McKenzie, Director), University of California, Berkeley, Calif., 1967.

4. B. Jónsson, Topics in universal algebra, Lecture Notes, Vanderbilt University, Nashville, Tenn., 1969/70.

5. R. S. Pierce, Introduction to the theory of abstract algebras, Holt, Rinehart and Winston, New York, 1968. MR 37 \#2655.

6. M. Yoeli and A. Ginzburg, On homomorphic images of transition graphs, J. Franklin Inst. 278 (1964), 291-296. MR 29 \#5240.

Department of Mathematics, University of Illinois at Chicago Circle, Chicago, ILLINOIS 60680 